\title{
Childhood Phyllodes Tumour of the Breast
}

Patrick Kafui Akakpo, Edmund Muonir Der*, Paul Cooper and Jehoram T. Anim

Korle-bu Teaching Hospital, Ghana

\begin{abstract}
Phyllodes tumours are rare biphasic breast lesions that generally occur in older women than fibroadenomas. We report case of a 14 year old girl who presented with a 1 year history of an ulcerated fungating left breast mass that required mastectomy. The general lack of awareness of breast disease in our community or knowledge of available therapies is discussed. The relevant literature is also reviewed.
\end{abstract}

Keywords: Childhood; Phyllodes tumour; Breast cancer; Ghana

\section{Introduction}

Phyllodes tumours are uncommon lesions that account for about $1 \%$ of all primary breast tumours [1]. The average age of occurrence of phyllodes tumour is 45 years. Although rare in children a case has been documented in a six year old girl [2].

Histologically phyllodes tumour of the breast is a biphasic tumour resembling fibroadenoma, but with hypercellular intralobular mesenchymal component causing distortion of ductal epithelial structures. They are classified into benign, borderline, and malignant histotypes based on the microscopic features of the stromal component. This classification is useful in determining appropriate management. There appears to be no difference in occurrence of the different histological types with age and the behaviour of the tumours is similar irrespective of the age. Histologically benign tumours have less than 4 mitoses per 10 high power fields, have a mildly cellular stroma but also lack stromal overgrowth while borderline tumours have 4-9 mitoses per 10 high power fields, have a markedly cellular stroma and lack stromal overgrowth. Tumours that have more than nine mitoses per 10 high power fields, have marked stromal cellularity with stromal overgrowth are classified as malignant [3].

In Ghana, little is known about the occurrence of this tumour and especially in adolescents and young girls. Because of the uncommon nature of this tumour in children some cases may be misdiagnosed and managed wrongly, resulting in recurrence and rarely, metastases. We report a case of borderline phyllodes tumour in a 14 year old girl and review the relevant literature.

\section{Case Report}

A 14 year old girl first presented to a local clinic with a mass in the left breast of one year duration. This had been managed at home with topical application of herbs but without improvement. The mass was described clinically as ulcerated, measuring $9 \times 15 \mathrm{~cm}$ and was not attached to the underlying muscle but had completely destroyed the nipple and areola. The skin immediately surrounding the ulcer was hypopigmented. The left axillary lymph nodes were enlarged but not matted together. They were not attached to the overlying or underlying tissues. The largest was $1 \mathrm{~cm}$ in diameter. No other lymph nodes were palpable. The right breast was grossly normal. All other systems of the body were normal. Chest radiograph, full blood count, urine routine examination and liver function tests were all normal. A simple mastectomy was done.

The specimen consisted of a grossly distorted breast that measured $15 \times 13 \times 9.5 \mathrm{~cm}$. The areola and nipple were replaced by a large fungating mass extending deep into the breast tissue (Figures 1and 2). Cut surface showed a lobulated solid light- brown coloured tumour. Parts of the

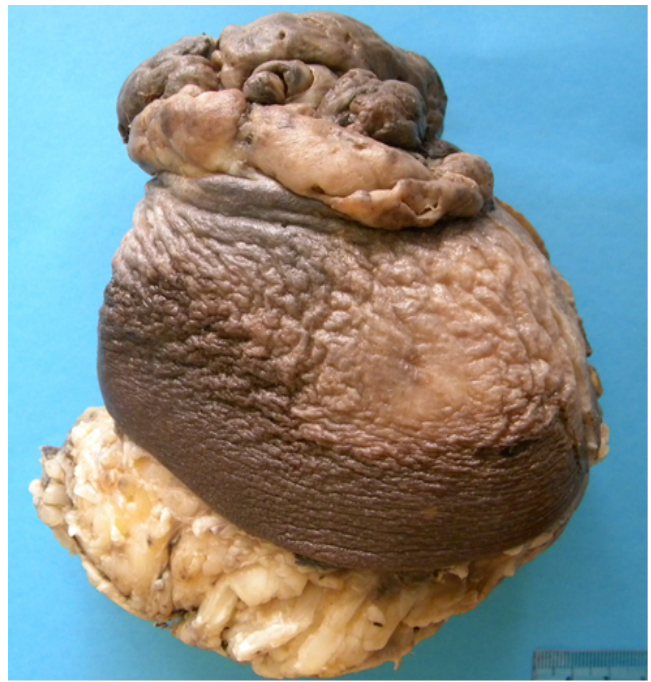

Figure 1: Showing the ulcerated breast with the fungating tumour replacing the nipple and areola and distorting the architecture of the entire breast. It also shows hypopigmentation of the skin around the base of the tumour.

tumour showed slit- like clefts. The lateral and deep margins were well circumscribed and the surrounding breast was normal. The tumour did not reach the deep and lateral margins of excision. No lymph nodes were received as part of the specimen.

Histologically there was ulceration of the skin by a tumour composed of proliferating spindle and polygonal cells. There were 5 mitoses per 10 high power fields and the tumour was interspersed with elongated irregularly shaped ductal structures lined by cuboidal to columnar epithelium which was multilayered in areas. The tumour was fairly well defined (Figure 3). There was a mild lymphoplasmacytic infiltrate within the tumour with increased numbers of neutrophils close to the ulcer. The tumour was $2 \mathrm{~cm}$ from the deep resection margin. The surrounding breast tissue showed normal ducts and stroma. A diagnosis of borderline phyllodes tumour of the left breast was made.

*Corresponding author: Edmund Muonir Der, Korle-bu Teaching Hospital, Box 77 Accra, Ghana, Tel: +233 - (243) 407809; E-mail: maadelle@yahoo.com

Received December 26, 2014; Accepted February 09, 2015; Published February 12, 2015

Citation: Akakpo PK, Der EM, Cooper P, Anim JT (2015) Childhood Phyllodes Tumour of the Breast. J Clin Case Rep 5: 495. doi:10.4172/2165-7920.1000495

Copyright: ( 2015 Der EM, et al. This is an open-access article distributed under the terms of the Creative Commons Attribution License, which permits unrestricted use, distribution, and reproduction in any medium, provided the original author and source are credited. 


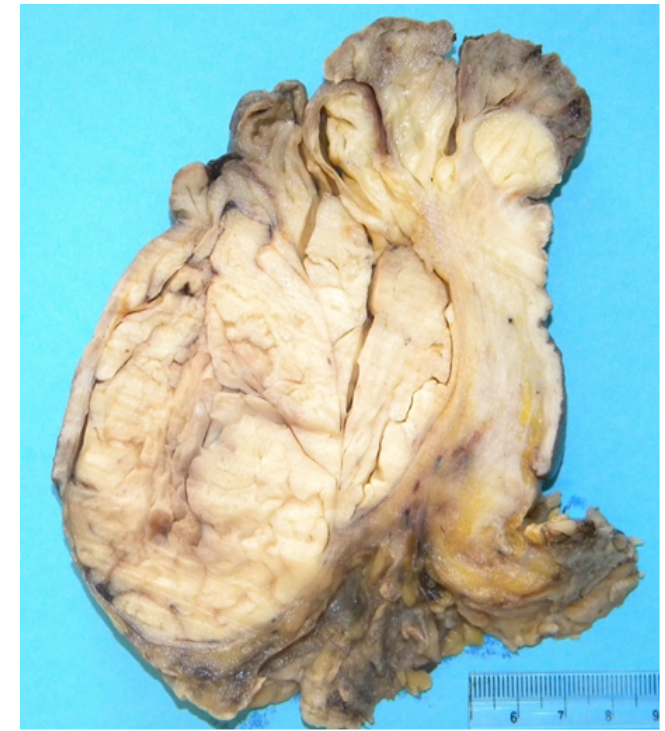

Figure 2: Section showing the cut surface of breast with almost complete replacement of the normal breast by a lobulated tumour with cleft-like spaces between the lobules. The cut surface of the fungating portion presents of the tumour also shows a leaf-like appearance.

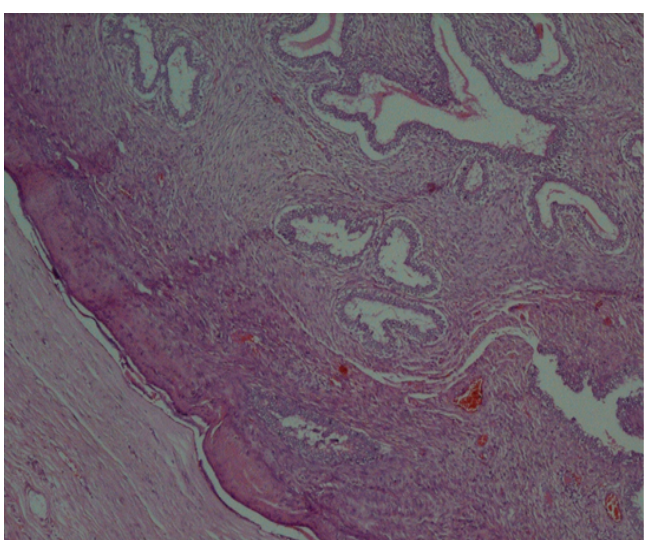

Figure 3: A photomicrograph of the edge of the tumour showing a dense cellular stroma and ductal structures lined by cuboidal to columnar epithelium bordering a densely collagenized normal stroma. The margin of the tumour (arrow) is well defined though no clear capsule is seen (H\&E; 100X).

\section{Discussion}

Phyllodes tumours are rare, accounting for less than $1 \%$ of mammary tumours [1] The median age of occurrence is 45 years, 15 years older than fibroadenomas, which are more common in younger women [4]. The age of the patient presented in this case report is 14 -years, which although young is within the age range reported in other publications outside Ghana that quoted a range of 10 to 24 years [5].

The patient presented with a large tumour (measuring $9 \times 15$ $\mathrm{cm}$ ) with ulceration of the overlying skin. Histologically, it had features of a borderline malignancy, hence the diagnosis. When they affect adolescents, Phyllodes tumours had been described as large. The large ulcerated breast tumour presented here is in accord with other publications on this type of breast disease [5]. In Ghana late presentation of women with breast cancers to health facilities has been reported [6-8]. The reasons for the late presentation include the fact that the lesions were painless (75\%), patient's expectation that the lesion might disappear with time (15\%) and a wait and see attitude (10\%) [9]. It is not surprising therefore, that this 14year-old girl also presented late to hospital with a large ulcerated breast mass.

Phyllodes tumour usually presents as a painless lump and our patient may have delayed in reporting to the hospital for any of the reasons stated above. General awareness of breast cancer in rural Ghana is poor and resort to alternative therapy is still rife among Ghanaians. The use of herbs may be due to the belief that they cause the mass to disappear. While using herbs a wait and see approach is adopted. The ulceration of the breast in this girl could be due to the herbs used and or the incision or scarification of such masses by traditional healers to allow herbs and other concoctions access into the swelling.

Assessments by clinical, radiological (mammographic) and cytological (FNA) or histological examinations form the recommended fundamental basis for evaluation of all breast lumps [3]. Accurate diagnosis and classification of phyllodes tumour is essential before surgery to help plan and ensure adequate excision with negative surgical margins [3]. This may be achieved through core biopsy. In our patient, except for clinical examination none of the other investigations commonly used to evaluate breast masses (imaging, cytology and histology) were used prior to surgery, most likely because of the sheer size of the lesion and the ulceration. In patients with Phyllodes tumours, all three methods individually have low sensitivity and even in combination, the diagnostic accuracy is often poor. This difficulty is because their clinical, imaging, cytology and histologic findings are often similar to those of fibroadenoma, which is a more common benign tumour [3]. Excision of the lump is often necessary for definitive diagnosis. In a study of 45 cases of phyllodes tumour in adolescents aged 10 to 24 years with tumours measuring $1.4 \mathrm{~cm}$ to $10.2 \mathrm{~cm}$ in their widest diameter, it was concluded that both breasts were affected equally and that phyllodes tumour in adolescent girls and young women is not more aggressive than in older patients [5]. At present there are no data on the incidence and awareness of phyllodes tumour in adolescents and children in Ghana, but in general, patients with breast disease tend to present late to the hospital, the duration varying from 2 days to 3 years in one study [9]. The mean time interval between onset of symptoms and presentation to the doctor is 3 months for breast lumps.

The reported frequency of local recurrence in incompletely resected tumours varies from 8 to $46 \%$ and age, tumour size, the surgical approach used, number of mitoses, extent of stromal overgrowth and safe surgical margins have been reported as predictive factors related to local recurrence [3]. Safety of surgical margins appears to be the most important among these and independently predicts improved diseasefree survival and decreased local recurrence [10]. The frequency of local recurrence is very low in cases with $1 \mathrm{~cm}$ or wider margins [11]. Mastectomy will in theory be the most reliable procedure with regard to local control, but breast-conservation surgery is preferred, except for very large tumours as in the present case. The surgical approach in this patient may have been informed by the size of the tumour relative to breast volume and the extent of destruction of the breast. Pathological examination showed that the tumour was $2 \mathrm{~cm}$ from the deep excision margin. Thus the choice of a mastectomy was appropriate. In incompletely excised cases, re-excision is recommended if there is stromal over growth and other obvious features of malignancy. Benign and borderline cases have a lower local recurrence rate because of the generally well circumscribed margins and it has been proposed that a 'wait-and-watch' policy is acceptable in these cases even when they 
appear to be incompletely excised $[12,13]$. The role of radiation therapy for phyllodes tumours remains unclear $[10,13]$.

Distant metastasis rates of up to $17 \%$ have been reported, mostly from borderline or malignant tumours. Many suggested histological prognostic factors for distant metastases have been evaluated, including stromal overgrowth, infiltrating margins, mixed mesenchymal components, high mitotic rate and stromal atypia [3]. All of these were absent in this patient. Although there were clinically palpable lymph nodes within the left axilla they were not submitted for pathologic examination. However lymphatic spread is not a known feature of phyllodes tumour and the enlargement of axillary lymph nodes in this patient may be due to the ulceration and secondary bacterial infection.

\section{Conclusion}

In conclusion awareness of breast diseases in adolescents and young children in Ghana needs to be improved through education. This report shows that phyllodes tumour can affect children and adolescents in Ghana and just as with cancer of the breast, greater awareness would ensure early presentation to hospital for appropriate treatment. In this particular case the 14 year-old girl may have been spared a mastectomy.

\section{References}

1. Tan PH, Tse G, Lee A, Simpson J, Hanby A (2012) Fibroepithelial tumours. WHO classification of Tumours of the breast. IARC Press, Lyon.

2. Mitsunori S, Tadaoki M, Kunio I, Hideo T, Yoshitaka K, et al. (1995) A malignant phyllodes tumour of the breast in a 6-year old girl Breast cancer J 2: 71-75
3. Naruto T, Daisuke T, Kenjiro A, Shozo O, Shigemitsu T, et al. (2007) Phyllodes Tumour of the Breast: Stromal Overgrowth and Histological Classification are Useful Prognosis-predictive Factors for Local Recurrence in Patients with a Positive Surgical Margin. Jap J Clin Oncol 37: 730-736

4. Abdelkrim S B, Trabelsi A, Bouzrara M, Boudagga M Z, Memmi A, et al. (2010) Phyllodes tumour of the Breast: A Review of 26 Cases. World J Oncol 3: 129134

5. Rajan PB, Cranor ML, Rosen PP (1998) Cystosarcoma phyllodes in adolescent girls and young women: a study of 45 patients. Am J Surg Pathol 22: 64-69.

6. EM Der, JN Clegg-Lamptey, RK Gyasi, JT Anim (2013) Positive malignant margins in clinically diagnosed and excised benign breast lumps: a five year retrospective study at the Korle-Bu teaching hospital, Ghana. J Med Biomed Sci 2: $21-25$

7. Edmund DM, Naaeder SB, Tettey Y, Gyasi RK (2013) Breast cancer in Ghanaian women: what has changed? Am J Clin Pathol 140: 97-102.

8. Der EM, Naaeder SB, Clegg-Lamptey JNA, Quayson SE, Wiredu EK (2014) Positive tumour margins in wide local excisions (lumpectomies) biopsies: a 10year retrospective study. Afr J Pathol and Microbiol.

9. Ohene-Yeboah M, Amaning E (2008) Spectrum of complaints presented at a specialist breast clinic in kumasi, ghana. Ghana Med J 42: 110-113.

10. Pandey M, Mathew A, Kattoor J, Abraham EK, Mathew BS, et al. (2001) Malignant phyllodes tumor. Breast J 7: 411-416.

11. Mangi AA, Smith BL, Gadd MA, Tanabe KK, Ott MJ, et al. (1999) Surgical management of phyllodes tumors. Arch Surg 134: 487-492.

12. Chua CL, Thomas A, Ng BK (1989) Cystosarcoma phyllodes: a review of surgical options. Surgery 105: 141-147.

13. Eich PD, Diederich S, Eich HT, Micke O, Wagner W (2000) Diagnostic radiation oncology: malignant cystosarcoma phylloides. Strahlenther Onkol 176: 192 195. 